\title{
Crash Risk Analysis for Shanghai Urban Expressways: a Bayesian Semi-parametric Modelling Approach
}

\author{
Rongjie Yua ${ }^{\mathrm{a}}$ Ph.D. \\ ${ }^{a}$ School of Transportation Engineering, Tongji University \\ 4800 Cao'an Road, 201804, Shanghai, China \\ Xuesong Wang ${ }^{\mathrm{a}^{*}}$, Ph.D. \\ ${ }^{a}$ School of Transportation Engineering, Tongji University \\ 4800 Cao'an Road, 201804, Shanghai, China \\ * Corresponding Author \\ wangxs@tongji.edu.cn \\ Kui Yang ${ }^{\mathrm{a}}$, Ph.D. Student \\ ${ }^{a}$ School of Transportation Engineering, Tongji University \\ 4800 Cao'an Road, 201804, Shanghai, China \\ Mohamed Abdel-Aty ${ }^{\mathrm{b}}$, Ph.D. \\ ${ }^{\mathrm{b}}$ Department of Civil, Environmental and Construction Engineering, \\ University of Central Florida Orlando, FL 32826-2450, United States
}

Nov 2015

Revision Submitted for possible publication in Accident Analysis \& Prevention 


\title{
Crash Risk Analysis for Shanghai Urban Expressways: a
}

\section{Bayesian Semi-parametric Modelling Approach}

\begin{abstract}
Urban expressway systems have been developed rapidly in recent years in China; it has become one key part of the city roadway networks as carrying large traffic volume and providing high travelling speed. Along with the increase of traffic volume, traffic safety has become a major issue for Chinese urban expressways due to the frequent crash occurrence and the non-recurrent congestions caused by them. For the purpose of unveiling crash occurrence mechanisms and further developing Active Traffic Management (ATM) control strategies to improve traffic safety, this study developed disaggregate crash risk analysis models with loop detector traffic data and historical crash data. Bayesian random effects logistic regression models were utilized as it can account for the unobserved heterogeneity among crashes. However, previous crash risk analysis studies formulated random effects distributions in a parametric approach, which assigned them to follow normal distributions. Due to the limited information known about random effects distributions, subjective parametric setting may be incorrect. In order to construct more flexible and robust random effects to capture the unobserved heterogeneity, Bayesian semi-parametric inference technique was introduced to crash risk analysis in this study. Models with both inference techniques were developed for total crashes; semi-parametric models were proved to provide substantial better model goodness-of-fit, while the two models shared consistent coefficient estimations. Later on, Bayesian semi-parametric random effects logistic regression models were developed for weekday peak hour crashes, weekday non-peak hour crashes, and weekend non-peak hour crashes to investigate different crash occurrence scenarios. Significant factors that affect crash risk have been revealed and crash mechanisms have been concluded.
\end{abstract}

Key words: Bayesian inference; Semi-parametric model; Crash risk analysis; Random effects logistic regression model

\section{Introduction}

With the rapid development of Chinese cities, residents' trip lengths have significantly increased while the trip modes have changed substantially from non-motorized to motorized. Along with the longer and motorized trips, the fast increase of vehicle ownership has resulted in more frequent traffic congestions. For the purpose of solving the congestion issue and providing higher traveling speeds, starting from the 1990s, Chinese cities began to construct the urban expressway systems. As defined in the design standards, urban expressways featured controlled access, more than four lanes (two directions), and divided (Ministry of Construction, 1990). By the year of 2011, Shanghai had a total mileage of $193 \mathrm{~km}$ urban expressways in operation. 
Along with the fast development of urban expressways in China, traffic volume on urban expressways increased dramatically. In 2011, the urban expressways in Shanghai have taken up $38.5 \%$ of the total traffic (only $5 \%$ of the total roadway mileage) (Shanghai urban construction and Transportation Commission, 2011). In addition, according to the Roadway Traffic Accident Statistical Annual Report (Ministry of Public Security Traffic Management Bureau, 2007), crashes occurred on urban expressways have accounted for $14.51 \%, 17.66 \%$, and $14.23 \%$ of the annual roadway traffic crashes with respective to total, fatal, and injury crashes, respectively in 2006. Considering the large traffic demand and frequent crash occurrence, it is essential to provide smoother and safer driving conditions for urban expressway users.

Metropolitans in the US and Europe recently utilize Active Traffic Management (ATM) Systems to manage their urban expressways proactively. However, in order to effectively prevent crash occurrence through control strategies such as Variable Speed Limits (VSL) and Queue Warning (QW), it is vital to understand the crash mechanisms on urban expressways. For the purpose of investigating crash contributing factors on urban expressways in Shanghai, this study conducts disaggregate traffic safety analyses based on historical crash data and traffic flow data captured by loop detectors. Crash risk analysis models were estimated for total crashes, weekday peak/non-peak hour crashes, and weekend non-peak hour crashes; Bayesian random effects logistic regression models were utilized to identify the relationships between traffic flow characteristics and crash risk.

One key part of the Bayesian random effects logistic regression models is the inference of the random effects term. Although previous studies focused on developing safety performance functions using random parameter models have tested various random effects distribution (Tulu et al., 2015; Xu and Huang, 2015), previous crash risk analysis studies subjectively assigned normal distributions with vague prior information (zero means and large variances) for random effects, which may be incorrect or unable to capture the unobserved heterogeneity. In order to construct more flexible and robust random effects distributions, Bayesian semi-parametric inference approach was adopted in this study. Modeling results and goodness-of-fits were compared between the parametric setting and semi-parametric approach.

The following parts of the paper were divided into five sections. Firstly, previous studies related to crash risk analysis and its modeling techniques, such as Bayesian logistic regression models and Bayesian semi-parametric models, were discussed. The second section provides a brief description of the data preparation procedures, followed by a description of the methodologies employed in this study. The fourth section presents the modelling results and discussion about the crash mechanisms on urban expressways at different time periods. Finally, summaries and conclusions of the work were given. 


\section{Background \\ Crash risk analysis}

Crash risk analysis was developed to identify crash occurrence precursors through investigating different traffic patterns between crash and non-crash scenarios; different methods have been utilized to formulate them. Among the various analysis methods, logistic regression models were the most frequently adopted one. Abdel-Aty et al., (2004) employed the matched case-control logistic regression modeling technique to analyze crash risk based on traffic flow data from loop detectors on I-4 in Orlando, Florida. Later on, Abdel-Aty and Pemmanaboina (2006) further incorporated weather data along with traffic data to assess the real-time crash risk on the same roadway with logistic regression models. Xu et al., (2012) utilized conditional logistic regression models with traffic data from I-880 in California to identify the traffic flow characteristics' effects on crash risk. Apart from the logistic regression models, data mining analysis methods such as neural network (2006) and support vector machine (Yu and Abdel-Aty, 2013a; Sun et al., 2014) were also employed to analyze crash risk. Although data mining approaches provided comparative or even superior goodness-of-fits as the logistic regression models did; the analysis procedure is argued to be a black-box which is not useful for crash occurrence mechanism investigation (Roshandel et al., 2015).

Recently, as the Bayesian inference technique became popular in traffic safety analysis studies due to its flexible model structure and the advantage of incorporating prior knowledge, Bayesian logistic regression models were developed to evaluate the crash risk on both freeway (Ahmed et al., 2012a) and urban expressway (Ahmed et al., $2012 b$ ). And it was claimed that the Bayesian analysis approach provided better fit and reduced uncertainty for parameter estimations (Ahmed et al., 2012b). In addition, in order to account for the unobserved heterogeneity between crashes, Bayesian random effects logistic regression models were applied (Xu et al., 2013; Yu and Abdel-Aty, 2013b; Yu et al., 2013); better model goodness-of-fits have been achieved. In this study, Bayesian random effects logistic regression models were developed to analyze crash occurrence mechanisms for urban expressways in China.

\section{Bayesian Semi-parametric models}

The Bayesian inference technique treats parameters as random variables which are characterized by prior distributions, which is the major difference with the Frequentist inference approach (Ntzoufras, 2009). Within the Bayesian inference framework, parametric Bayesian inference assumes the prior distributions can be indexed by finite-dimensional parameters, which would limit the inference scope and affect the model flexibility (Müller and Quintana, 2004). For the purpose of enriching the model flexibility and robustness and avoid mis-specifying parametric models, Bayesian semi-parametric or nonparametric models were introduced (Jara et al., 2011). Shively et al., (2010) adopted a Bayesian semi-parametric approach to analyze the 
relationships between crash counts and roadway characteristics. The nonparametric estimation procedure was employed for the model's link function and the results showed that the semi-parametric model was more robust compared to the standard parametric assumption.

One key advantage of Bayesian semi-parametric models is to model random effects distributions in hierarchical models (Müller and Quintana, 2004). Random effect terms were frequently introduced to account for the unobserved heterogeneity in crash risk analysis as mentioned above; since the true forms or distributions of these terms are unknown, semi-parametric approach is more reasonable for model estimation. However, to the best of our knowledge, no semi-parametric model has been adopted in crash risk analysis models. This study fills the gap as introducing semi-parametric Bayesian models to analyze crash risk and comparing the modeling results with parametric Bayesian models.

\section{Data Preparation}

Data from the Shanghai urban expressway system were utilized; the roadway system includes Yan'an elevated road, North-South elevated road, Middle ring elevated road, Inner ring elevated road, and Yixian elevated road. As shown in Figure 1, the Yan'an elevated road has an east-west trajectory, the North-South elevated road is north-south, and Yixian elevated road is radial; the Inner ring and the Middle ring elevated roads are two loops that cover mostly the urban areas of Shanghai. Three datasets were used to form the data: (i) crash data in January and March of 2009; (ii) roadway segment geometry data obtained from Shanghai Electronic Information Science Research Institute; (iii) traffic data detected by 1,646 LDs at 518 detector sections (the LDs are lane specified) located on both directions along Shanghai urban expressways. LD data provide lane-based average speed and total volume at 5-minute interval. 


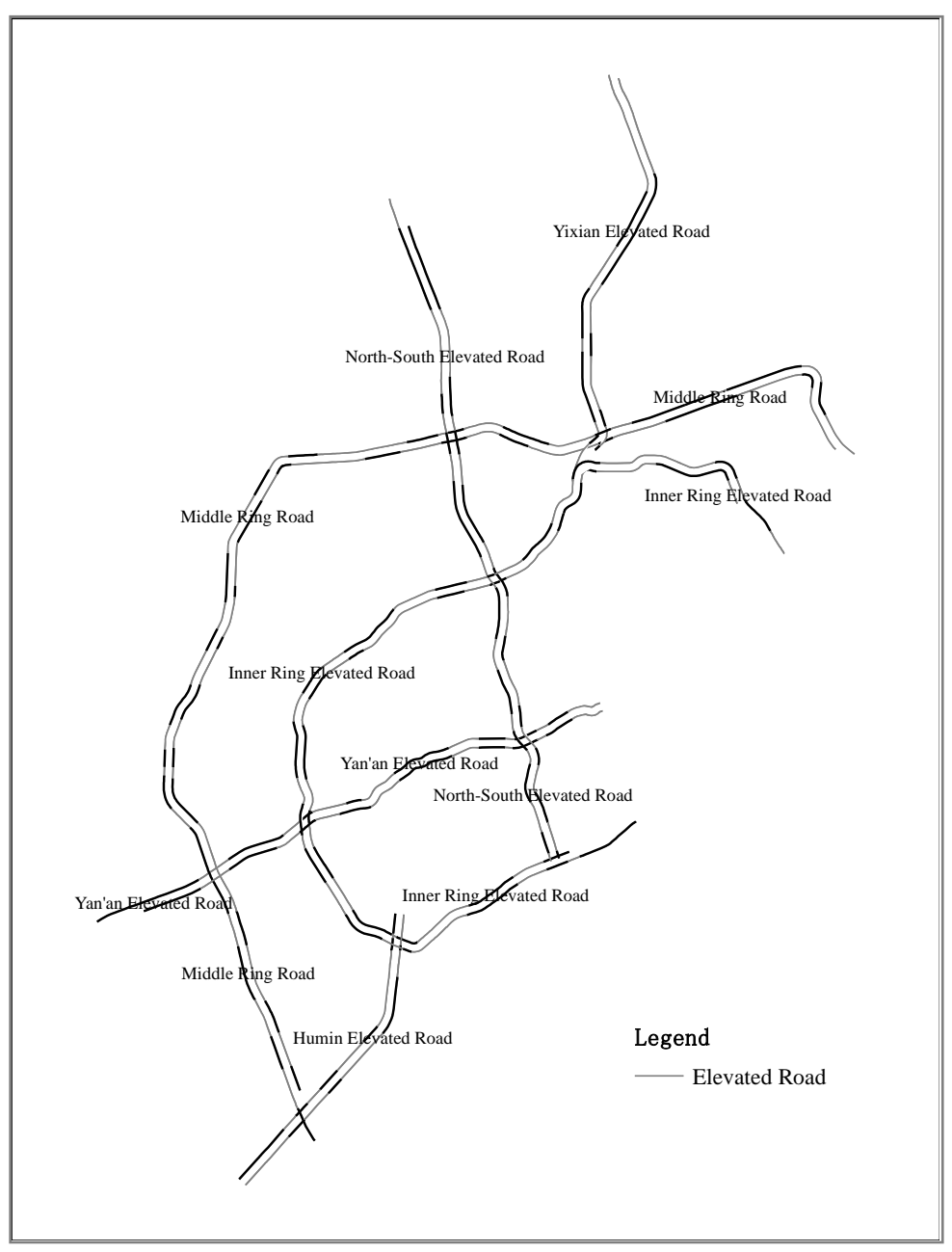

Figure 1 Shanghai urban expressway network

For each specific crash, stake number was utilized to describe its location (stake numbers are marked along the Shanghai urban expressway; generally consisted of letters or Chinese characters and numbers, and is ordered with non-repetitive numbers of designed foundation piles when constructed). The stake numbers of LDs on Shanghai urban expressway were collected for the purpose of identifying and matching traffic data with crash data; ArcMap (ESRI, 2006) were employed to identify the upstream and downstream LDs for each crash.

Based on the location of each crash, four nearest detector stations (two stations upstream and two stations downstream) were identified to monitor the traffic conditions prior to crash occurrence. The first upstream and downstream stations were named as U1 and D1, respectively; and the farthest stations in the upstream and downstream direction were named as $\mathrm{U} 2$ and D2, respectively. The arrangements of stations are shown in Figure 2. 


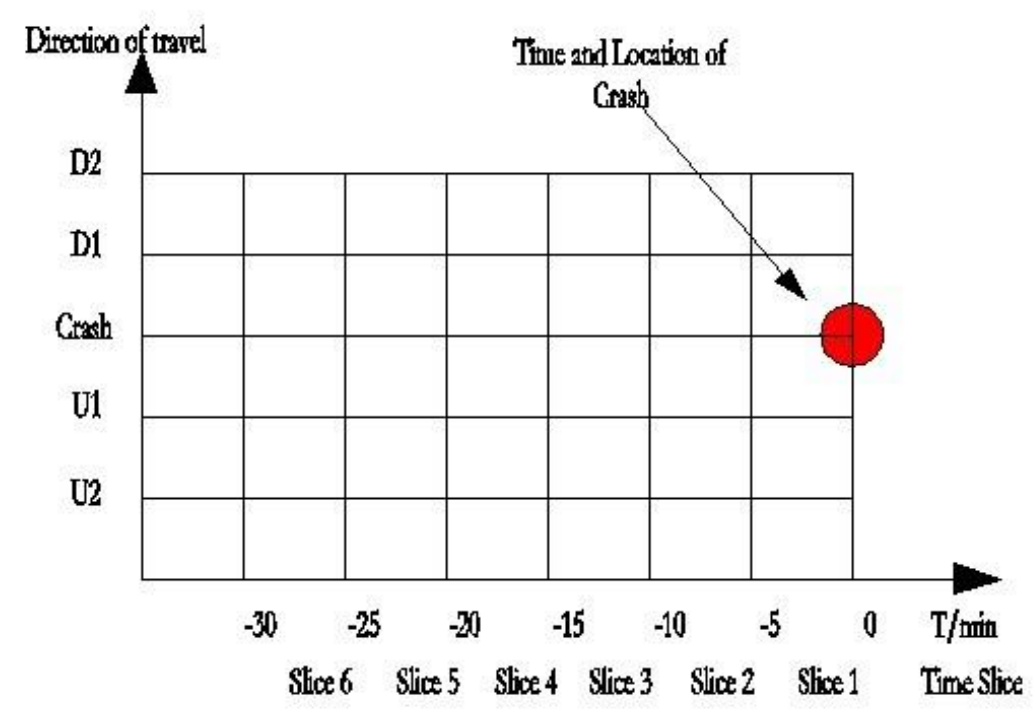

Figure 2 Nomenclature for defining stations and time-slices relative to location and time of crash

For a crash occurred on March 28, 2009 (Saturday) at 8:40 p.m., traffic data from 08:10 p.m. to 08:40 p.m. (30 minutes window) were extracted and named as time-slices 1, 2, 3, 4, 5, and 6 (as shown in Figure 2), with slice 1 being the 5 minutes window before the crash reporting time. Based on the traffic data availability, a total of 2567 crash records were used in the following analysis.

In addition, for the intents of comparing normal traffic conditions with traffic characteristic prior to crash occurrence, non-crash cases' traffic data were also extracted. For each crash, 4 non-crash cases were included. The final dataset has 2,567 matched strata with 2,567 crashes and 8,448 non-crashes (the non-exact 1:4 crash and non-crash ratio is due to the LD data availability issue).

These non-crash traffic conditions were collected from selected combinations of time and location where no crash was observed within the 2-hour window, given the same time of day, day of week, month and location but in different weeks. For example, a crash that occurred on NN0312 (stake number) on March 28, 2009 at 8:40 p.m., the traffic conditions in the same place and time in March 21, March 14, March 7 were collected as controls (non-crash cases).

Average speed, total volume, and standard deviation of speed and volume between lanes based on detector station were calculated at 5-min interval and included as explanatory variables in the crash risk analysis model. The nomenclature of variables includes 3 letters and 2 numbers. The first letter takes the value A, S, or T for average, standard deviation, and total, respectively. While the second letter takes the value of $S$ or $\mathrm{V}$ for speed and volume, respectively. The group of the third letter and the initial 
number takes the value of $\mathrm{U} 2, \mathrm{U} 1, \mathrm{D} 1, \mathrm{D} 2$ representing the station to which the traffic parameters belong. The last number takes the value of 1,2, 3, 4, 5 and 6 which refer to the six time slices used in the study. The nomenclature of variable is shown as Figure 3. In addition, in order to avoid the mis-recording crash time issue, variables from time slice 1 were dropped for final analysis.

\section{$* * * * *$}

Average/Standard deviation/Total (AS/T)

Speed/Volume(S/V)
Time slice (1/2/3/4/5/9)

Detector station (U2/U1/D1/D2)

Figure 3 Nomenclature of variable

In addition, in order to analyze crashes occurred within different time periods, average speeds and total volumes for the urban expressway system were utilized to identify the peak and non-peak hours for weekday and weekend respectively. As shown in Figure 4, peak hours were identified as 7:00-9:00 and 16:00-18:00 for weekdays and 9:30-11:30 and 15:00-18:00 for weekends; other time periods were regarded as non-peak hours. Furthermore, crash counts for different time periods are given in Table 1.

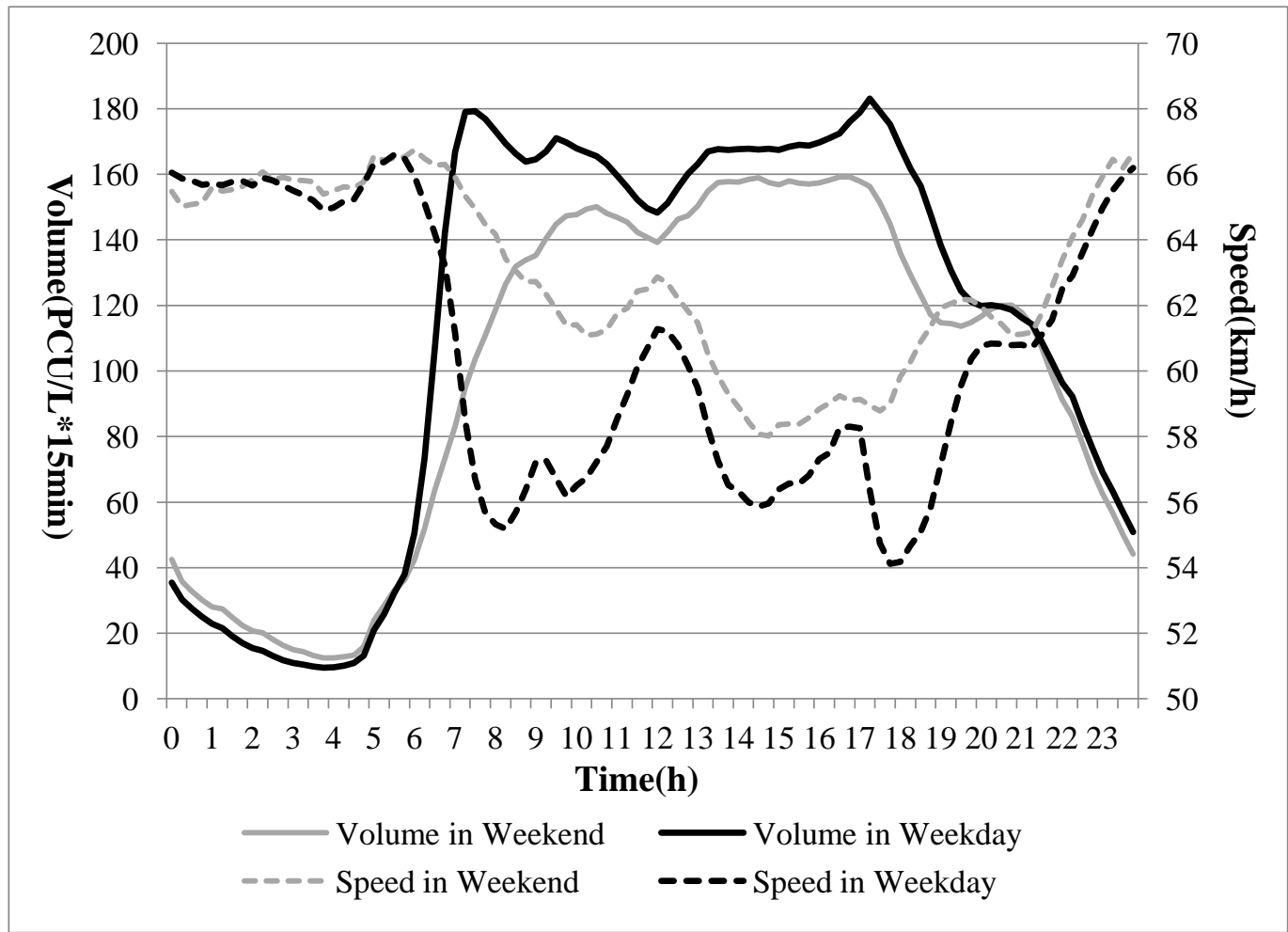

Figure 4 Distributions of volume and speed for weekdays and weekends

Table 1 Average daily crash counts for different time periods

\begin{tabular}{|c|c|}
\hline Time Period & $\begin{array}{c}\text { Average Daily } \\
\text { Crash Counts }\end{array}$ \\
\hline
\end{tabular}




\begin{tabular}{|l|l|c|}
\multirow{4}{*}{ Weekday } & Non-Peak & 285 \\
\cline { 2 - 3 } & Morning Peak & 46 \\
\cline { 2 - 3 } & Evening Peak & 56 \\
\hline \multirow{3}{*}{ Weekend } & Non-Peak & 192 \\
\cline { 2 - 3 } & Morning Peak & 37 \\
\cline { 2 - 3 } & Evening Peak & 86 \\
\hline
\end{tabular}

\section{Methodology}

Two different types of model were employed in this study: parametric Bayesian random effects logistic regression model and semi-parametric Bayesian random effects logistic regression model. This section will first introduce the random effects logistic regression model formulation and then illustrate the different settings for the parametric random effects term and semi-parametric random effects term.

Suppose crash occurrence has the outcomes of $\mathrm{y}=1$ (crash case) and $\mathrm{y}=0$ (non-crash case) with respective probability $p$ and $1-p$. The random effects logistic regression can be setup as follows:

$$
\begin{gathered}
y \sim \text { Binomial }(p) \\
\operatorname{logit}(p)=\log \left(\frac{p}{1-p}\right)=\boldsymbol{X} \boldsymbol{\beta}+u_{j}(i)
\end{gathered}
$$

where $\boldsymbol{X}$ is the vector of the explanatory variables, $\boldsymbol{\beta}$ is the coefficients vector for the explanatory variables. The coefficients of the explanatory variables were set up with non-informative priors as following normal distributions (Normal $(0,1000)) . u_{j}$ is the random effects variable defined in the model, which represents the crash level random error. $i$ stands for the observation number and $j$ indicates the number of matched strata for observation $i$.

For the parametric Bayesian inference approach, a total of six functional forms were considered for the random effects term, which are normal, lognormal, exponential, Weibull, uniform and triangular as tested in the previous literature (Anastasopoulos and Mannering, 2011; Xu and Huang, 2015). Based on the statistical fits, normal distribution outperformed the others, which was then used in the following developed parametric random effects Bayesian logistic regression models.

For the semi-parametric approach, Bush and MacEachern (1996) proposed a Dirichlet processes (DP) prior for the random effects term while Kleinman and Ibrahim (1998) adopted the same approach in a more general framework. In addition, Müller and Rosner (1997) utilized a Bayesian population model with hierarchical mixture priors to analyze blood count data. The mixture model was specified a hyperprior distribution by means of a DP prior on the mixing measure. It was claimed that the proposed model was capable of addressing most non-normal and non-linear features. In this study, a Mixture of Dirichlet processes (MDP) prior was utilized. 
The MDP prior replace the assumption of a parametric prior with a general distribution $G$. The distribution G was assigned to have a DP prior,

$$
\begin{gathered}
u_{j} \mid \mathrm{G} \sim \mathrm{G} \\
\mathrm{G} \mid \alpha, G_{0} \sim D P\left(\alpha G_{0}\right) \\
\alpha \mid a_{0}, b_{0} \sim \operatorname{Gamma}\left(a_{0}, b_{0}\right) \\
G_{0}=N(\theta \mid \mu, \Sigma) \\
\mu \sim \operatorname{Normal}\left(\mu_{b}, S_{b}\right) \\
\Sigma \mid v_{0}, T \sim \operatorname{IW}\left(v_{0}, T\right)
\end{gathered}
$$

where $G_{0}$ is a j-dimensional parametric distribution and $\alpha$ is a positive scalar. The parameter $G_{0}$ is called as the base measure, which is a distribution that approximates the true nonparametric shape of G. The scalar $\alpha$ represents the prior belief about how similar the nonparametric distribution $\mathrm{G}$ is to the base measure $G_{0}$.

For the model estimation, parametric Bayesian models were estimated through WinBUGS (Lunn et al., 2000). For each model, three chains of 15,000 iterations were set, 5,000 iterations were used in the burn-in step. While for the semi-parametric Bayesian models, DPpackage (Jara et al., 2011) in R (R, 2012) was utilized ${ }^{1}$. DIC, which is recognized as Bayesian generalization of AIC (Akaike information criterion) and it was adopted for model comparisons. DIC is a combination of model fit measurement and the effective number of parameters; the smaller DIC indicates a better model fit. According to Spiegelhalter et al. (2003), differences of more than 10 might definitely rule out the model with higher DIC. Differences between 5 and 10 are considered substantial. Besides, AUC value, which is areas under the receiver operating characteristic (ROC) curve, was chosen to evaluate and compare these models; larger AUC values indicate a better goodness-of-fit and classification power.

\section{Modeling Results}

In order to unveil the crash mechanisms, crash risk models were firstly developed with Bayesian random effects logistic regression models utilizing both parametric and semi-parametric inference techniques for total crashes. Modeling results were compared based on goodness-of-fits and forecasting accuracy measurements. Furthermore, for the purpose of identifying various crash occurrence scenarios at different time periods, a likelihood-ratio test (LRT) has been conducted in the revised version to support the separate estimation of models by time-of-day. The LRT is given by:

$$
-2\left[L L\left(\beta_{T}\right)-L L\left(\beta_{w d p}\right)-L L\left(\beta_{w d n p}\right)-L L\left(\beta_{w e p}\right)-L L\left(\beta_{w e n p}\right)\right]
$$

where $\operatorname{LL}\left(\beta_{T}\right)$ is the log-likelihood at convergence of the model estimated with total crash data, $L L\left(\beta_{w d p}\right)$ is the log-likelihood at convergence for weekday peak hours

\footnotetext{
${ }^{1}$ Note, in this study the semi-parametric and parametric models were developed using different statistical software.
} 
crash data, and $L L\left(\beta_{w d n p}\right)$ represents the log-likelihood at convergence for weekday non-peak hours crash data, and $L L\left(\beta_{\text {wep }}\right)$ and $L L\left(\beta_{\text {wenp }}\right)$ are the log-likelihoods for weekend peak hours crash data and weekend non-peak hours crash data respectively. The likelihood ratio test has a $x^{2}$ value of 116.3 with 10 degrees of freedom, which indicates that the crash data should be analyzed by time-of-day at $99 \%$ confidence level. The LRT analysis result further suggests that possible differences of crash risk contributing factors are not entirely captured by the variables included in the total crash model. Therefore, crash risk affecting factors were investigated for weekday peak hours, weekday non-peak hours, and weekend non-peak hours; due to the small sample sizes of weekend peak hour crashes, model for weekend peak hours were not considered here.

The developed models were based on the calculated 80 baseline variables ( 2 statistics $\times 2$ traffic flow variables $\times 4$ detector stations $\times 5$ time slices), a backward variable selection approach was first conducted. Manual variable selections and variable transformations were then utilized to identify the final significant variables. Finally, the multicollinearity issue was checked before the final models.

\section{Total crash model}

For total crash models, four variables were found to be significantly associated with crash occurrence; correlations between the variables have been checked. Table 2 shows summary statistics for the variables while

Table 3 lists the modeling results for the Bayesian random effects logistic regression models.

Table 2 Summary statistics for significant variables

\begin{tabular}{llllll}
\hline Variable & Definition & Mean & Std. Dev & Min & Max \\
\hline ASU12 & Average Speed for detector U1 & 47.67 & 22.53 & 0 & 100.15 \\
ASD12 & Average Speed for detector D1 & 49.63 & 20.40 & 0 & 108.4 \\
SVD12 & Std Dev of Volume for detector D1 & 18.37 & 15.40 & 0 & 157.28 \\
TVD22 & Total Volume for detector D2 & 288.69 & 149.19 & 0 & 870.0 \\
\hline
\end{tabular}

Table 3 Modeling results for total crashes

\begin{tabular}{lcccc|cccc}
\hline & \multicolumn{4}{c|}{ Parametric random effects } & \multicolumn{3}{c}{ Semi-parametric random effects } \\
& \multicolumn{3}{c}{ Bayesian logistic regression model } & \multicolumn{3}{c}{ Bayesian logistic regression model } \\
\hline Variable & Mean & Std Dev & $2.5 \%$ & $97.5 \%$ & Mean & Std Dev & $2.5 \%$ & $97.5 \%$ \\
\hline Intercept & -0.13 & 0.08 & -0.29 & 0.02 & -0.14 & 0.084 & -0.30 & 0.028 \\
ASU12 & -0.015 & 0.001 & -0.017 & -0.012 & -0.0147 & -0.0012 & -0.017 & -0.012 \\
ASD12 & -0.004 & 0.001 & -0.007 & -0.0016 & -0.004 & 0.0014 & -0.007 & -0.0016 \\
SVD12 & 0.0026 & 0.001 & -0.0001 & 0.005 & $0.0027 *$ & 0.0014 & -0.0001 & 0.005 \\
TVD22 & -0.0008 & 0.0001 & -0.001 & -0.0004 & -0.0008 & 0.0001 & -0.001 & -0.0004 \\
\hline
\end{tabular}




\begin{tabular}{|c|c|c|c|c|c|c|}
\hline$\tau$ & 0.003 & 0.0015 & 0.006 & \multicolumn{3}{|c|}{ N/A } \\
\hline$\mu$ & \multicolumn{2}{|r|}{ N/A } & -0.15 & 0.54 & -1.25 & 0.89 \\
\hline$\Sigma$ & \multicolumn{2}{|r|}{ N/A } & 0.37 & 0.23 & 0.078 & 1.48 \\
\hline$\alpha$ & \multicolumn{2}{|r|}{ N/A } & 0.49 & 0.40 & 0.05 & 1.56 \\
\hline DIC & \multicolumn{2}{|r|}{11687.4} & \multicolumn{4}{|c|}{11678.2} \\
\hline AUC & \multicolumn{2}{|r|}{0.61} & \multicolumn{4}{|c|}{0.70} \\
\hline MAD & \multicolumn{2}{|r|}{0.348} & \multicolumn{4}{|c|}{0.335} \\
\hline RMSE & \multicolumn{2}{|r|}{0.174} & \multicolumn{4}{|c|}{0.169} \\
\hline \multicolumn{3}{|c|}{ No. of Observations } & 11015 & & & \\
\hline
\end{tabular}

* Significant at $90 \%$ level

From the results it can be seen that the two models provided similar modeling outcomes; same variables were identified and consistent estimated coefficient signs were given. The four significant variables are all within the time slice of 5-10 minutes prior to crash occurrence. Among the four variables, the two average speed variables (ASU12 and ASD12) both have negative coefficients. Both upstream and downstream of the crash location have slow moving traffic, which indicates that crashes are more likely to occur within congested roadway segments. This analysis result is consistent with previous crash risk analysis studies (Garber and Wu, 2001; Park and Oh, 2009; Christoforou et al., 2011). In addition, crash risk is more influenced by the upstream operating speed. The SVD12 variable is significant with a positive sign; larger volume variation will increase crash occurrence likelihood. This can be interpreted as that increase of volume variations between lanes will lead to more opportunities of lane changing maneuvers, which will increase the sideswipe and rear-end crash risk. In addition, the 5-mintue total volume at detector D2 is significant with a negative sign; lower traffic volume will increase crash possibility. Considering the low operating speed at D1 location, low volume at D2 will cause vehicles around D1 speed up to leave the slow moving platoon; vehicles accelerate at different levels will increase rear-end crash probability.

For the model comparison, based on the DIC values it can be concluded that the semi-parametric model provides a substantial better model fit compared to the parametric model (11678.2 vs. 11687.4). Besides, indicating by the AUC values, the semi-parametric model has improved the overall model fitting by $14.75 \%$. Moreover, both Mean Absolute Deviation (MAD) and Root Mean Square Error (RMSE) comparison results provided support that semi-parametric model hold better forecasting accuracy. Considering the comparable coefficient estimations, the better goodness-of-fit was achieved from the non-parametric setting for the random effects term. From the random effects estimations it can be seen that the semi-parametric approach random effects distribution have larger variance compared to the parametric approach and the scalar parameter $\alpha$ provides more flexible distribution formulations. Therefore, since the semi-parametric model outperformed the parametric model, semi-parametric inference approach was adopted for different time periods crash risk 
analysis.

\section{Weekday peak hour crash model}

The urban expressway system suffered from recurrent congestion during weekday peak hours due to the large traffic demands, which lead to continuously stop-and-go flow. In order to unveil the typical crash occurrence scenarios and design suitable ATM control strategies to resolve the recurrent congestions, crash risk analysis for weekday peak hours was conducted. Three variables were found to be significant; Table 4 shows the summary statistics and Table 5 lists the modeling results.

Table 4 Summary statistics for significant variables

\begin{tabular}{|c|c|c|c|c|c|}
\hline Variable & Definition & Mean & Std. Dev & Min & Max \\
\hline SVU12 & $\begin{array}{l}\text { Std Dev of Volume for detector } \\
\text { U1 for time slice } 2\end{array}$ & 17.19 & 15.02 & 0 & 125.18 \\
\hline DiffASU1_34 & $\begin{array}{l}\text { Average Speed Difference for } \\
\text { detector U1 between time slice } 3 \\
\text { and } 4\end{array}$ & 0.72 & 6.78 & -42.5 & 53.8 \\
\hline ASU13 & $\begin{array}{l}\text { Average Speed for detector U1 } \\
\text { for time slice } 3\end{array}$ & 44.30 & 23.08 & 30 & 86.82 \\
\hline
\end{tabular}

Table 5 Modeling results for weekday peak hours crashes

\begin{tabular}{|c|c|c|c|c|}
\hline \multirow[b]{2}{*}{ Variable } & \multicolumn{4}{|c|}{$\begin{array}{c}\text { Semi-parametric random effects Bayesian logistic } \\
\text { regression model }\end{array}$} \\
\hline & Mean & Std Dev & $2.5 \%$ & $97.5 \%$ \\
\hline Intercept & -0.84 & 0.12 & -1.08 & -0.61 \\
\hline SVU12 & 0.007 & 0.003 & 0.0008 & 0.014 \\
\hline DiffASU1_34 & 0.034 & 0.007 & 0.019 & 0.049 \\
\hline ASU13 & -0.012 & 0.002 & -0.016 & -0.007 \\
\hline$\mu$ & -0.85 & 0.44 & -1.72 & 0.02 \\
\hline$\Sigma$ & 0.29 & 0.44 & 0.058 & 1.07 \\
\hline$\alpha$ & 1.78 & 3.92 & 0.088 & 12.15 \\
\hline DIC & \multicolumn{4}{|c|}{2325.9} \\
\hline AUC & \multicolumn{4}{|c|}{0.713} \\
\hline No. of Observations & \multicolumn{4}{|c|}{6016} \\
\hline
\end{tabular}

Three variables were found to affect crash risk, which are all from upstream traffic detectors. SVU12 has a positive coefficient, which indicates that as the traffic volume variation between lanes increase, the crash occurrence probability will increase. Large traffic volume variation between lanes will cause more lane change maneuver, which will lead to more exposure for sideswipe and rear-end crashes. Two speed variables are significant where ASU13 has a negative sign and DiffASU1_34 has a positive sign. With the estimated coefficients of ASU13 and DiffASU1_34 it can be inferred that before the crash occurrence, upstream travel speed changed from high speed to 
low speed during time slice 4 (10-15 min before crash occurrence) and time slice 3 (15-20 min before crash occurrence), the speed reduction may lead to frequent stop-and-go conditions which will increase the risk of rear-end crashes.

\section{Weekday non-peak hour crash model}

Unlike the weekday peak hour crashes which were affected by the upstream traffic flow, final significant factors (Table 6) showed that crashes occurred during weekday non-peak hours are more related to downstream traffic statuses. From Table 7 it can be seen that ASU12 and ASD12 are both significant with negative signs, which demonstrate that crashes are more likely to occur within congested roadway segments. TVD22 holds a negative coefficient; large traffic volume at D2 will reduce crash risk. DiffTVD2_23 holds a positive coefficient indicates that there is a distinct traffic volume drop from time slice 3 to time slice 2 at location D2 before crash occurrence, which would cause upstream traffic to speed up and increase the rear-end crash probability. Besides, the weekday non-peak hours model shared three similar significant variables with the total crash model, this may be due to that crashes occurred during weekday non-peak hours take up a large proportion of the total crashes (from Table 1 it can be seen that weekday non-peak hours crashes have taken up $55.6 \%$ of the total crash sample).

The typical crash scenario is that for the crash occurrence prone locations there is a slow moving traffic platoon; while at far downstream locations, the slow moving traffic start to dissipate. The evanescent wave moves upstream which lead to chaos around the crash locations; whereas crash risk enhanced significantly.

Table 6 Summary statistics for significant variables

\begin{tabular}{llllll}
\hline Variable & Definition & Mean & Std. Dev & Min & Max \\
\hline ASU12 & Average Speed for detector U1 & 47.20 & 22.80 & 0 & 100.15 \\
TVD22 & Total Volume for detector D2 & 292.06 & 151.82 & 0 & 870 \\
DiffTVD2_23 & $\begin{array}{l}\text { Total Volume Difference for } \\
\text { detector D2 }\end{array}$ & 0.27 & 35.96 & -321 & 387 \\
& Average Speed for detector D1 & 49.03 & 20.5 & 0 & 99.68 \\
\hline
\end{tabular}

Table 7 Modeling results for weekday non-peak hours crashes

\begin{tabular}{c|llll}
\hline & \multicolumn{4}{|c}{$\begin{array}{c}\text { Semi-parametric random effects Bayesian logistic } \\
\text { regression model }\end{array}$} \\
\hline Variable & Mean & Std Dev & $2.5 \%$ & $97.5 \%$ \\
\hline Intercept & -0.047 & 0.11 & -0.26 & 0.18 \\
ASU12 & -0.015 & 0.0016 & -0.018 & -0.012 \\
TVD22 & -0.0008 & 0.0002 & -0.0012 & -0.0004 \\
DiffTVD2_23 & 0.0024 & 0.0008 & 0.0007 & 0.004 \\
ASD12 & -0.004 & 0.0018 & -0.008 & -0.0006 \\
\hline
\end{tabular}




\begin{tabular}{|c|c|c|c|c|}
\hline$\mu$ & -0.058 & 0.53 & -1.11 & 0.99 \\
\hline$\Sigma$ & 0.36 & 0.57 & 0.076 & 1.44 \\
\hline$\alpha$ & 0.55 & 0.46 & 0.058 & 1.73 \\
\hline DIC & \multicolumn{4}{|c|}{6419.0} \\
\hline AUC & \multicolumn{4}{|c|}{0.742} \\
\hline No. of Observations & \multicolumn{4}{|c|}{6016} \\
\hline
\end{tabular}

\section{Weekend non-peak hour crash model}

Due to the small sample size of weekend peak hour crashes, crash risk contributing factors for weekend peak hours were not investigated in this study. For weekend non-peak hour crashes, three variables were determined to be significant; whereas Table 8 lists the summary statistics and Table 9 shows the modeling results. ASU12 is significant with a negative sign, which indicates that operating speeds upstream of the crash locations were relatively low before crash occurrence. SSU12 also holds a negative coefficient, which further confirms that the upstream traffic is congested for all lanes as there is not much difference for speed between lanes. LogTVD22 has a negative coefficient; the detector D2 has low traffic volume. From the modeling results it can be assumed that weekend non-peak hour crashes occurred where the upstream location was congested while the far down stream is a free-flow condition. Vehicles near the crash locations started to speed up and change lanes to achieve their desired speeds, the disordered traffic flow would increase the crash occurrence likelihood.

Table 8 Summary statistics for significant variables

\begin{tabular}{|c|c|c|c|c|c|}
\hline Variable & Definition & Mean & Std. Dev & Min & $\operatorname{Max}$ \\
\hline ASU12 & Average Speed for detector U1 & 54.58 & 19.23 & 0 & 94.30 \\
\hline SSU12 & $\begin{array}{l}\text { Std Dev of Speed for detector } \\
\text { U1 }\end{array}$ & 5.96 & 7.97 & 0 & 62.93 \\
\hline $\log T V D 22$ & $\begin{array}{l}\text { Logarithm transformation of } \\
\text { Total Volume for detector D2 }\end{array}$ & 5.36 & 0.72 & 0 & 6.59 \\
\hline
\end{tabular}

Table 9 Modeling results for weekend non-peak hours crashes

\begin{tabular}{|c|c|c|c|c|}
\hline \multirow[b]{2}{*}{ Variable } & \multicolumn{4}{|c|}{$\begin{array}{c}\text { Semi-parametric random effects Bayesian logistic } \\
\text { regression model }\end{array}$} \\
\hline & Mean & Std. Dev & $2.5 \%$ & $97.5 \%$ \\
\hline Intercept & 0.84 & 0.49 & -0.19 & 1.78 \\
\hline ASU12 & -0.018 & 0.003 & -0.024 & -0.013 \\
\hline SSU12 & -0.02 & 0.008 & -0.039 & -0.006 \\
\hline $\log \mathrm{TVD} 22$ & -0.18 & 0.08 & -0.34 & -0.02 \\
\hline$\mu$ & 0.82 & 0.62 & -0.43 & 2.0 \\
\hline$\Sigma$ & 0.25 & 0.34 & 0.056 & 0.99 \\
\hline$\alpha$ & 2.72 & 4.04 & 0.11 & 14.88 \\
\hline
\end{tabular}

DIC

1725.2




\section{Summary and Conclusion}

Urban expressways have become the key part of the city roadway systems for Chinese cities. Non-recurrent congestions caused by traffic crashes are significantly affecting operating efficiency. For the purpose of unveiling crash occurrence mechanisms, this study was carried out to investigate crash occurrence precursors through developing crash risk analysis models. As proposed and suggested in previous studies, Bayesian random effects logistic regression model was utilized here. One key inference interest of the Bayesian random effects logistic regression model is the posterior estimation of the random effects. Previous studies mostly adopted a parametric approach as assigning normal distributions to the random effects term. In order to better capture the uncertainty about the exact distribution of the random effects term, different distribution types and semi-parametric inference technique was introduced.

Models were developed for total crashes and crashes occurred in weekday peak hours, weekday non-peak hours, and weekend non-peak hours. Due to the small sample size, weekend peak hour model were not considered. Crash occurrence contributing factors and scenarios were concluded and typical crash scenarios can be drawn from the modeling results. For total crashes, there is a congested area around the crash location while downstream has large volume variation between lanes. Vehicles suffering from the congestion dissipation wave will have a larger probability of being involved in crashes. Unlike the total crashes associated with both upstream and downstream traffic flow characteristics, crashes occurring during weekday peak hours are more impacted by upstream traffic. Variations of volume and the speed drops would increase crash occurrence likelihood. In addition, as the weekday non-peak hour crashes take up a large proportion of the total crashes, the identified crash mechanisms were similar to those concluded for total crashes. Congestion dissipation waves passed from downstream locations would increase the collision probability of vehicles travelling within slow moving platoons. Moreover, crashes that happened during weekend non-peak hours shared similar crash scenarios with weekday non-peak hours crash but different significant contributing factors were concluded.

The benefits of utilizing Bayesian random effects logistic regression models to analyze crash risk have been proved in the previous studies. However, settings of random effects term were subjective, which may fail to account for the population heterogeneity and restrict the model flexibility. This study takes a step forward as utilizing semi-parametric inference technique for the random effects estimation. For the modeling comparisons, consistent coefficient estimations have been concluded; semi-parametric models have substantial lower DIC values, which demonstrate better goodness-of-fit and forecasting accuracy. In addition, based on the estimation results 
for the random effects distributions it can be concluded that the parametric setting of zero mean normal distributions are not suitable for specific datasets (for weekday peak hour crashes, the random effects distribution has significantly negative mean estimations).

A previous study (Pande and Abdel-Aty, 2006) has concluded that geometric characteristics have impacts on crash risk; therefore, the geometric characteristics in different crash occurrence locations would contribute to the heterogeneity between crashes. Through the random effects logistic regression models proposed in this study, the unobserved heterogeneity caused by geometric characteristics would be captured. Furthermore, based on the modeling results, appropriate ATM control strategies can be proposed to address the traffic safety issue for Shanghai urban expressway systems. For weekday peak hours crashes, they suffered from congestions propagating from downstream; queue warning systems can be utilized to provide downstream congestion information to upstream drivers. Besides, for crashes occurred during the non-peak hours, Variable Speed Limits system can be configured to slow down the congestion platoon dissipation process.

Acknowledgement: This study was co-sponsored by the Chinese National Science Foundation (71401127) and the Science and Technology Commission of Shanghai Municipality, China (15DZ1204800). Dr.Abdel-Aty conducted this research in collaboration with Tongji University while funded by SAFER-SIM, a tier 1 lead by the University of Iowa. 


\section{Reference}

Abdel-Aty, M., Uddin, N., Pande, A., Abdalla, F., and Hsia, L., 2004. Predicting freeway crashes from loop detector data by matched case-control logistic regression. Transportation Research Record 1897, 88-95.

Abdel-Aty, M. and Pemmanaboina, R., 2006. Calibrating a real-time traffic crash-prediction model using archived weather and ITS traffic data. IEEE Transactions on Intelligent Transportation Systems 7, 167-174.

Ahmed, M., Abdel-Aty, M., and Yu, R., 2012. Assessment of the Interaction between Crash Occurrence, Mountainous Freeway Geometry, Real-Time Weather and AVI Traffic Data. Transportation Research Record 2280, 51-59.

Ahmed, M, Abdel-Aty, M., and Yu, R., 2012. A Bayesian Updating Approach for Real-Time Safety Evaluation Using AVI Data. Transportation Research Record 2280, 60-67.

Anastasopoulos, P., Mannering, F., 2011. An empirical assessment of fixed and random parameter logit models using crash- and non-crash-specific injury data. Accident Analysis and Prevention, 43(3), 1140-1147.

Bush, C., and MacEachern, S., 1996. A semiparametric Bayesian model for randomised block designs. Biometrika 83, 275-285.

ESRI, 2006. ArcMap 9.2. ESRI Inc.

Jara, A., Hanson, T., Quintana, F., Müller, P., and Rosner, G., 2011. DPpackage: Bayesian semi-and nonparametric modeling in R. Journal of Statistical Software 40(5).

Kleinman, K, and Ibrahim, J., 1998. A semiparametric Bayesian approach to the random effects model. Biometrics, 921-938.

Lunn, D., Thomas, A., Best, N., and Spiegelhalter, D., 2000. WinBUGS-a Bayesian modelling framework: concepts, structure, and extensibility. Statistics and Computing 10, 325-337.

Ministry of Construction of the People's Republic of China., 1990. City road design standard. No. CJJ37-90.

Ministry of Public Security Traffic Management Bureau., 2007. Roadway Traffic Accident Statistical Annual Report.

Müller, P., and Quintana, F., 2004. Nonparametric Bayesian data analysis. Statistical Science, 95-110.

Müller, P., and Rosner, G., 1997. A Bayesian population model with hierarchical mixture priors applied to blood count data. Journal of the American Statistical Association 92, 1279-1292.

Pande, A., and Abdel-Aty, M., 2006. Comprehensive analysis of the relationship between real-time traffic surveillance data and rear-end crashes on freeways. Transportation Research Record 1953, 31-40.

Ntzoufras, I., 2009. Bayesian modeling using WinBUGS. WILEY

R: A language and environment for statistical computing. 2012.

Roshandel, S., Zheng, Z., and Washington, S., 2015. Impact of real-time traffic characteristics on freeway crash occurrence: Systematic review and meta-analysis. Accident Analysis and Prevention 79, 198-211. 
Shanghai urban construction and Transportation Commission., 2011. "Twelfth Five Year" development planning of comprehensive transportation in Shanghai City.

Shively, T., Kockelman, K., and Damien, P., 2010. A Bayesian semi-parametric model to estimate relationships between crash counts and roadway characteristics. Transportation Research Part B: Methodological 44, 699-715.

Spiegelhalter, D., Thomas, A., Best, N., and Lunn, D., 2003. WinBUGS user manual. Cambridge: MRC Biostatistics Unit.

Sun, J., Sun, J., and Chen, P., 2014. Using support vector machine models for real-time crash risk prediction on urban expressways. In 2014 TRB Annual Meeting, Washington D.C.

Tulu, S., Washington, S., Haque, M., and King, M., 2015. Investigation of pedestrian crashes on two-way two-lane rural roads in Ethiopia. Accident Analysis and Prevention, 78, 118-126.

Xu, C., Liu, P., Wang, W., and Li, Z., 2012. Evaluation of the impacts of traffic states on crash risks on freeways. Accident Analysis and Prevention 47, 162-171.

Xu, C., Wang, W., and Liu, P., 2013. Identifying crash-prone traffic conditions under different weather on freeways. Journal of Safety Research 46, 135-144.

Xu, P., Huang, H., 2015. Modeling crash spatial heterogeneity: Random parameter versus geographically weighting. Accident Analysis and Prevention, 75, 16-25.

Yu, R., and Abdel-Aty, M, 2014. An optimal variable speed limits system to ameliorate traffic safety risk. Transportation Research Part C: Emerging Technologies 46, 235-246.

Yu, R., and Abdel-Aty, M, 2013a. Utilizing support vector machine in real-time crash risk evaluation. Accident Analysis and Prevention 51, 252-259.

Yu, R., and Abdel-Aty, M, 2013b. Multi-level Bayesian analyses for single-and multi-vehicle freeway crashes. Accident Analysis and Prevention 58, 97-103.

Yu, R., Abdel-Aty, M. and Ahmed, M., 2013. Bayesian random effect models incorporating real-time weather and traffic data to investigate mountainous freeway hazardous factors. Accident Analysis and Prevention 50, 371-376. 